\title{
The Contents of Phytosterols, Squalene, and Vitamin E and the Composition of Fatty Acids of Korean Landrace Setaria italica and Sorghum bicolar Seeds
}

\author{
Shiva Ram Bhandari and Young-Sang Lee ${ }^{1}$ * \\ Vegetable Research Division, National Institute of Horticultural and Herbal Science, Rural Development \\ Administration, Suwon 440-706, Korea \\ ${ }^{1}$ Department of Medical Biotechnology, Soonchunhyang University, Asan 336-745, Korea
}

\begin{abstract}
To characterize the nutraceutical property of Italian millet (Setaria italica) and sorghum (Sorghum bicolor), ten Korean landraces of each crop were collected and their vitamin E (tocopherols and tocotrienols), squalene and phytosterols (campesterol, stigmasterol and $\beta$-sitosterol) contents as well as fatty acid composition in seeds were evaluated. Italian millet seeds exhibited 5 forms of vitamin E isomers: three ( $\alpha-, \gamma-$ and $\delta-)$ tocopherols and two ( $\alpha-$ and $\gamma-$ ) tocotrienols, while sorghum seeds showed only three forms of vitamin $E$ isomers: $\alpha$ - and $\gamma$-tocopherol and $a$-tocotrienol. In both crops, $\gamma$ -tocopherol was the major constituent of vitamin $\mathrm{E}$ in terms of highest quantity. Total vitamin E content in Italian millet and sorghum landraces were $88.3 \mathrm{mg} / \mathrm{kg}$ and $44.3 \mathrm{mg} / \mathrm{kg}$, respectively. Among three phytosterols (campesterol, stigmasterol and $\beta$-sitosterol) analyzed, $\beta$-sitosterol was the major form comprising about $85 \%$ and $65 \%$ in Italian millet and sorghum landraces, respectively. Total phytosterols content ranged from 443.0 to $568.5 \mathrm{mg} / \mathrm{kg}$ and 442.3 to $719.2 \mathrm{mg} / \mathrm{kg}$ in Italian millet and sorghum, respectively. Squalene, a precursor of phytosterols biosynthesis, ranged from 6.8 to $10.2 \mathrm{mg} / \mathrm{kg}$ in Italian millet and from 62.2 to $115.2 \mathrm{mg} / \mathrm{kg}$ in sorghum. Linoleic, oleic and palmitic acids were the major fatty acids in both of the crops and about $80 \%$ of the total fatty acids were unsaturated fatty acids. Among the tested landraces, M09 and S10 showed relatively higher proportion of phytonutrients, suggesting their potential as a gene source for further breeding program.
\end{abstract}

Key words - Phytonutrient, Cereal, Tocotrienol, Tocopherol, Campesterol, Sitosterol, Stigmasterol

\section{Introduction}

Italian millet (Setaria italica) and Sorghum (Sorghum bicolor) are world's sixth and fifth most important cereal grains, respectively, in terms of both production and cultivation area (FAO, 2011). They are inexpensive and nutritionally comparable or even superior to major cereals (Pathak et al., 2000; Duodu et al., 2003). These crops are staple foods that supply a major proportion of calories and protein to large segments of populations in the semi-arid tropical regions of Africa and Asia (O'Kennedy et al., 2006). The Italian millet and sorghum can also grow and give higher and more stable grain yields under poor soil and growing conditions.

In addition to starch, a major constituent of cereal crop

${ }^{\star}$ Corresponding author. E-mail : mariolee@sch.ac.kr seeds, the presence of various phytochemicals such as vitamins, polyphenols, flavonoids, minerals, phytosterols as well as fatty acids enrich the nutritional value of cereals. And consequently it is noteworthy to trace such health beneficial phytochemicals in seeds of cereal crops. Vitamin E, squalene, and phytosterols are health beneficial compounds present in the unsaponifiable lipid fraction of cereal crops. Vitamin E consisting of four tocopherols ( $\alpha-, \beta-, \gamma-$, and $\delta$-tocopherol) and the corresponding tocotrienols ( $\alpha-, \beta-, \gamma-$, and $\delta$-tocotrienol) is a fat soluble antioxidant and functions as scavengers of lipid peroxyl radicals. Tocopherol content in food is inversely associated with mortality from cardiovascular disease (Knekt et al., 1994; Kushi et al., 1996). In addition, tocopherols, due to their capacity to quench free radical damage, play a putative role in prevention of Alzheimer's disease and cancer (Tucker and Townsend, 2005). Among the four tocopherol isomers, a- 
tocopherol is considered as the most biologically active form (Ohkatsu et al., 2001) and has the function of a radical-chain breaking antioxidant in membranes and lipoproteins, as well as in foods (Kamal-Eldin and Appelqvist, 1996). Tocotrienols, another form of vitamin $\mathrm{E}$ have been reported to exhibit health-beneficial effects similar to tocopherols; antioxidative, antiproliferative (Choi and Lee, 2009), anticancer (Wada et al., 2005), and cholesterol biosynthesis-inhibiting effects (Qureshi et al., 1995). Phytosterols, primarily $\beta$-sitosterol, campesterol, and stigmasterol are integral natural components of plant cell membranes that are abundant in vegetable oils, nuts, and grains (Weihrauch and Gardner, 1978). They have a variety of biological effects including serum-cholesterol lowering effects (Ostlund, 2004; Marangoni and Poli, 2010), anti-inflammatory, anti-oxidative, and anti-carcinogenic activities (de Jong et al., 2003). Several studies have shown that plant sterols inhibit the intestinal absorption of cholesterol, thereby lowering total plasma cholesterol and low-density lipoprotein (LDL) levels (de Jong et al., 2003). Squalene, a 30 carbon isoprenoid, is a key intermediate in cholesterol biosynthesis (Moreda et al., 2001) and is an important dietary cancerchemopreventive agent (Smith, 2000). More recently, squalene has been shown to act as an antidote to reduce accidental drug-induced toxicities (Aguilera et al., 2005; Senthilkumar et al., 2006). The protective effect of squalene may be attributed to its ability to serve as an antioxidant; e.g., it has been demonstrated to be a potent quencher of singlet oxygen (Kohno et al., 1995) and protects against $\mathrm{H}_{2} \mathrm{O}_{2}$-induced sister chromatid exchange (SCE) in Chinese hamster V79 cells (O’Sullivan et al., 2002).

Most of phytonutrient studies on cereals, however, have been conducted intensively for major crops. There are several reports regarding the polyphenols, tannins and flavonoid content, antioxidant as well as antiradical activities and proximate nutrient composition in millet and sorghum (Awika and Rooney, 2004; Dykes and Rooney, 2006; Chethan and Malleshi, 2007). Information regarding lipophilic phytonutrients such as vitamin E, phytosterols, and fatty acid composition in these cereals is rare. Although Pirronen et al. (2002) and Ryan et al. (2007) have analyzed phytosterols in millet, only one variety had been selected and the variety name was not clearly identified. Singh et al. (2003) analyzed phytosterols in sorghum but the number of variety was only one. So, it will be noteworthy to analyze the lipophilic phytonutrients in various sorghum and millet landraces to select any landrace having higher content of phytonutrients for the breeding program. Hence, this study was mainly focused on evaluation of tocopherol, tocotrienol, squalene and phytosterol contents as well as fatty acid composition in seeds of Italian millet and sorghum landraces collected from Korea.

\section{Materials and Methods}

\section{Sample collection}

Grains of ten landraces of each sorghum and Italian millet were kindly donated from Sinlim Agricultural Cooperative Federation of Wonju City, Kangwon-Do, South Korea. The list of landraces and their Korean names are provided in Table 1. Grain samples delivered to analysis laboratory were ground to fine powders and stored at $-80^{\circ} \mathrm{C}$ prior to nutrient analyses conducted within 1 month after delivery.

\section{Chemicals and Reagents}

Authentic standards of squalene, campesterol, stigmasterol, and $\beta$-sitosterol were purchased from Sigma-Aldrich (St. Louis, MO, USA). Standards of FAME (fatty acid methyl ester) were acquired from Supelco (Bellefonte, PA, USA), and vitamin $E$ standards (tocopherols: $\alpha-, \beta-, \gamma-$, and $\delta$-tocopherol; and tocotrienols: $\alpha-, \beta-, \gamma$ - and $\delta$-tocotrienol) were purchased from Merck (Darmstadt, Germany). Ascorbic acid, chloroform

Table 1. The name and abbreviations of Italian millet and sorghum landraces used in this experiment

\begin{tabular}{|c|c|}
\hline Crop & Korean variety names and abbreviations of landraces \\
\hline Italian millet & $\begin{array}{l}\text { Hwangum Mejo (M01), Mejo (M02), Heuin Chajo (M03), Jangsoo Hwang Chajo (M04), Buksimie Chajo (M05), } \\
\text { Hwang Chajo (M06), Auroon Chajo (M07), Ohl Jo (M08), Ggojang Jo (M09), Neut Jo (M10) }\end{array}$ \\
\hline Sorghum & $\begin{array}{l}\text { Chal Susu (S01), Ilbanchal Susu (S02), Bulgeunjangmok Susu (S03), Bulgeunjangsu Susu (S04), Me Susu (S05), } \\
\text { Jangsu Susu (S06), Mongdang Susu (S07), Sikyung Susu (S08), Susongsaengie Susu (S09), Heun Susu (S10) }\end{array}$ \\
\hline
\end{tabular}


and anhydrous sodium sulfate were obtained from Samchun (Seoul, Republic of Korea), and benzene, ethanol, potassium hydroxide, sulfuric acid and n-heptane were purchased from Daejung (Seoul, Republic of Korea), and 2,2-dimethoxypropane was obtained from Sigma-Aldrich (St. Louis, MO, USA). Other chemicals, including $n$-hexane (HPLC grade), iso-octane (2,2,4-trimethyl pentane; HPLC grade) and methanol (HPLC grade) were purchased from J.T. Baker (Phillipsburg, NJ, USA).

\section{Vitamin E, squalene and phytosterols analysis}

The samples for the analyses of vitamin E isomers ( $\alpha-, \beta-$, $\gamma-$, and $\delta$-tocopherol and $\alpha-, \beta-, \gamma-$, and $\delta$-tocotrienol), phytosterols (campesterol, stigmasterol and $\beta$-sitosterol) and squalene were prepared and analyzed based upon the procedure previously described by Park et al. (2004) and Bhandari et al. (2012). Briefly, the powdered samples (1.0 g) were placed in a $50 \mathrm{~mL}$ tube, and $0.1 \mathrm{~g}$ ascorbic acid was added as an antioxidant along with $10 \mathrm{~mL}$ ethanol prior to shaking in a water bath at $80^{\circ} \mathrm{C}$ for $10 \mathrm{~min}$. Then, $300 \mu \mathrm{L}$ of $44 \% \mathrm{KOH}$ was added and the mixture was shaken for saponification for $18 \mathrm{~min}$ in a water bath at $80^{\circ} \mathrm{C}$. The tubes were cooled rapidly in an ice bucket, after with $10 \mathrm{~mL} \mathrm{n}$-hexane and $10 \mathrm{~mL}$ of distilled water were added, mixed, and centrifuged for $10 \mathrm{~min}$ at $1000 \mathrm{rpm}$; and the upper hexane layer was collected. This process was repeated three times, and the collected hexane layers were pooled and washed three times with $10 \mathrm{~mL}$ distilled water and passed through anhydrous $\mathrm{Na}_{2} \mathrm{SO}_{4}$ to remove water, concentrated in a rotary evaporator and dissolved in iso-octane. Then the samples were analyzed by gas chromatography (Varian 3800, Palo Alto, CA, USA). The analysis was performed with a capillary column $(\mathrm{CP}-\mathrm{SIL} 8 \mathrm{CB}, 30 \times 0.25 \mathrm{~mm}$, $0.4 \mu \mathrm{m}$ film thickness) with the injector and FID temperatures set at $290^{\circ} \mathrm{C}$. The injection volume was $1 \mu \mathrm{L}$ with a split ratio of 1:20 and the carrier gas $(\mathrm{He})$ flow rate was $1.0 \mathrm{~mL} / \mathrm{min}$. The oven temperature was initially set at $220^{\circ} \mathrm{C}$ for $2 \mathrm{~min}$, increased to $290^{\circ} \mathrm{C}$ by $5^{\circ} \mathrm{C} / \mathrm{min}$, held for $14 \mathrm{~min}$, and then increased to $310^{\circ} \mathrm{C}$ at a rate of $10^{\circ} \mathrm{C} / \mathrm{min}$. Peak identifications were conducted based upon the retention times of authentic standard compounds.

\section{Fatty acid composition analysis}

Samples for fatty acid composition analysis were prepared according to Kim et al. (2000) with slight modifications. Powdered samples $(0.2 \mathrm{~g})$ were mixed with $680 \mu \mathrm{L}$ of a methylation mixture (MeOH: benzene: 2,2-dimethoxypropane: $\mathrm{H}_{2} \mathrm{SO}_{4}=39: 20: 5: 2$ ) and $400 \mu \mathrm{L}$ of heptane. After vigorous mixing, the solution was heated for $2 \mathrm{hr}$ at $80^{\circ} \mathrm{C}$ in a water bath and cooled to room temperature. The heptane layer was collected by centrifugation and was injected into a gas chromatography (Varian, CP-3800, Palo Alto, CA, USA) equipped with a capillary column (CP-SIL 88 CB FAME, $50 \times 0.25 \mathrm{~mm}$, $0.2 \mu \mathrm{m}$ film thickness; Supelco, Bellefonte, PA, USA). The temperatures for injector and FID detector were set at $210^{\circ} \mathrm{C}$ and $290^{\circ} \mathrm{C}$, respectively and the carrier gas was helium. The injection volume was $1 \mu \mathrm{L}$ with a split ratio of 1:50 on constant column flow $(1.0 \mathrm{~mL} / \mathrm{min})$. Oven temperature was initially set on $100^{\circ} \mathrm{C}$ for $5 \mathrm{~min}$, then raised up to $180^{\circ} \mathrm{C}$ at $4^{\circ} \mathrm{C}$ /min increasing rate, held for 5 minutes and then increased to $210^{\circ} \mathrm{C}$ by $5^{\circ} \mathrm{C} / \mathrm{min}$, and held for $20 \mathrm{~min}$. A mixture of $37 \mathrm{FAME}$ standards was used to identify the peaks based upon the retention time. The relative percentage of each identified fatty acid was calculated based upon their peak area and used for the composition of each fatty acid.

\section{Data analysis}

Means of three independent sample replications were used and statistical analyses were performed with Duncan's multiple range tests using SPSS (version 18, SPSS, Inc., Chicago, IL, USA) at a significance level of $\mathrm{p}=0.05$.

\section{Results and Discussion}

\section{Vitamin E content}

Among 8 vitamin $\mathrm{E}$ isomer tested, 5 vitamin $\mathrm{E}$ isomers (3 tocopherols and 2 tocotrienols) could be quantified in Italian millet while only 3 vitamin $\mathrm{E}$ isomers (2 tocopherols and 1 tocotrienol) were observed in sorghum. In both of the crops, the major form of vitamin $\mathrm{E}$ isomer was $\gamma$-tocopherol which showed $63.1 \mathrm{mg} / \mathrm{kg}$ and $37.5 \mathrm{mg} / \mathrm{kg}$ in average of tested landraces corresponding to $71 \%$ and $85 \%$ of total vitamin $\mathrm{E}$ in Italian millet and sorghum, respectively (Table 2 and 3). In the case of Italian millet, total tocopherol content ranged from $66.3 \mathrm{mg} / \mathrm{kg}$ (M07) to $94.2 \mathrm{mg} / \mathrm{kg}$ (M01) with an average of $79.1 \mathrm{mg} / \mathrm{kg}$. Our findings for the tocopherols were higher 
Table 2. Tocopherol and tocotrienol contents in seeds of Italian millet landraces $(\mathrm{mg} / \mathrm{kg})$

\begin{tabular}{|c|c|c|c|c|c|c|c|c|}
\hline Landrace & a-tocopherol & $\gamma$-tocopherol & $\delta$-tocopherol & a-tocotrienol & $\gamma$-tocotrienol & $\begin{array}{c}\text { Total } \\
\text { tocopherol }\end{array}$ & $\begin{array}{c}\text { Total } \\
\text { tocotrienol }\end{array}$ & Total vitamin $\mathrm{E}$ \\
\hline M01 & $11.7^{\mathrm{z}} \mathrm{cd}^{\mathrm{y}}$ & $80.7 \mathrm{a}$ & $1.8 \mathrm{a}$ & $1.7 \mathrm{a}$ & $7.8 \mathrm{bcd}$ & $94.2 \mathrm{a}$ & $9.5 \mathrm{bcd}$ & $101.9 \mathrm{a}$ \\
\hline M02 & $13.9 \mathrm{bc}$ & $73.7 \mathrm{a}$ & $2.0 \mathrm{a}$ & $1.6 \mathrm{ab}$ & 7.6bcde & $89.5 \mathrm{ab}$ & $9.3 \mathrm{bcde}$ & $98.8 \mathrm{ab}$ \\
\hline M03 & $17.5 \mathrm{a}$ & $56.0 \mathrm{bc}$ & $1.4 \mathrm{a}$ & $1.7 \mathrm{ab}$ & $9.2 \mathrm{ab}$ & $75.0 \mathrm{~cd}$ & $10.7 \mathrm{ab}$ & $85.6 \mathrm{~cd}$ \\
\hline M04 & $19.3 \mathrm{a}$ & $56.8 \mathrm{~b}$ & $2.1 \mathrm{a}$ & $1.3 \mathrm{bcde}$ & $8.3 \mathrm{bcd}$ & $78.1 \mathrm{c}$ & $9.6 \mathrm{bcd}$ & $87.7 \mathrm{bc}$ \\
\hline M05 & $14.6 b$ & $62.0 \mathrm{~b}$ & $1.5 \mathrm{a}$ & $1.4 \mathrm{abcd}$ & $8.7 b c$ & $78.1 \mathrm{c}$ & $10.1 b c$ & $88.2 \mathrm{bc}$ \\
\hline M06 & $17.0 \mathrm{a}$ & $62.8 \mathrm{~b}$ & $1.3 \mathrm{a}$ & $1.1 \mathrm{de}$ & $6.8 \mathrm{def}$ & $81.0 \mathrm{~cd}$ & 7.9def & $88.9 \mathrm{bc}$ \\
\hline M07 & $17.3 \mathrm{a}$ & $48.7 \mathrm{c}$ & $0.3 \mathrm{a}$ & $1.0 \mathrm{e}$ & 7.4cdef & $66.3 \mathrm{~d}$ & $8.4 \mathrm{cdef}$ & $74.7 \mathrm{~d}$ \\
\hline M08 & $10.5 \mathrm{~d}$ & $64.2 \mathrm{~b}$ & $0.4 \mathrm{a}$ & $1.5 \mathrm{abcd}$ & $6.0 \mathrm{f}$ & $75.0 \mathrm{~cd}$ & $7.5 \mathrm{ef}$ & $82.6 \mathrm{~cd}$ \\
\hline M09 & $13.5 \mathrm{bc}$ & $64.0 \mathrm{~b}$ & $0.5 \mathrm{a}$ & $1.5 \mathrm{abc}$ & $10.4 \mathrm{a}$ & $77.9 \mathrm{c}$ & $11.9 \mathrm{a}$ & $89.9 \mathrm{bc}$ \\
\hline M10 & $12.5 \mathrm{bcd}$ & $62.6 \mathrm{~b}$ & $0.4 \mathrm{a}$ & $1.2 \mathrm{cde}$ & $6.2 \mathrm{ef}$ & $75.5 \mathrm{~cd}$ & $7.4 f$ & $82.9 \mathrm{~cd}$ \\
\hline Average & 14.8 & 63.1 & 1.2 & 1.4 & 7.8 & 79.1 & 9.2 & 88.3 \\
\hline
\end{tabular}

${ }^{7}$ Values are mean of three independent replications.

${ }^{\mathrm{y}}$ Means followed by different letters within a column are significantly different at $\mathrm{p}<0.05$ by Duncan's multiple range tests.

Table 3. Tocopherol and tocotrienol contents in seeds of sorghum landraces $(\mathrm{mg} / \mathrm{kg})$

\begin{tabular}{ccccccc}
\hline \hline Landrace & $\alpha$-tocopherol & $\gamma$-tocopherol & $\alpha$-tocotrienol & Total tocopherol & Total tocotrienol & Total vitamin E \\
\hline S01 & $4.5^{\mathrm{z}} \mathrm{ab}^{\mathrm{y}}$ & $43.0 \mathrm{ab}$ & $2.1 \mathrm{~b}$ & $48.4 \mathrm{ab}$ & $2.1 \mathrm{~b}$ & $50.6 \mathrm{ab}$ \\
S02 & $4.9 \mathrm{ab}$ & $44.4 \mathrm{a}$ & $1.8 \mathrm{bc}$ & $50.6 \mathrm{a}$ & $1.8 \mathrm{ab}$ & $52.5 \mathrm{a}$ \\
S03 & $3.9 \mathrm{~b}$ & $41.7 \mathrm{ab}$ & $1.5 \mathrm{~cd}$ & $46.9 \mathrm{abc}$ & $1.5 \mathrm{~cd}$ & $48.3 \mathrm{abc}$ \\
S04 & $4.6 \mathrm{ab}$ & $27.1 \mathrm{~d}$ & $1.3 \mathrm{a}$ & $31.9 \mathrm{a}$ & $1.3 \mathrm{~d}$ & $33.2 \mathrm{e}$ \\
S05 & $5.1 \mathrm{a}$ & $33.5 \mathrm{~cd}$ & $1.5 \mathrm{~cd}$ & $38.7 \mathrm{cde}$ & $1.5 \mathrm{~cd}$ & $40.2 \mathrm{cde}$ \\
S06 & $4.0 \mathrm{ab}$ & $36.8 \mathrm{bc}$ & $1.8 \mathrm{bc}$ & $41.3 \mathrm{bcd}$ & $1.8 \mathrm{bc}$ & $43.1 \mathrm{bcd}$ \\
S07 & $4.3 \mathrm{ab}$ & $32.5 \mathrm{~cd}$ & $3.6 \mathrm{a}$ & $37.0 \mathrm{de}$ & $3.6 \mathrm{a}$ & $40.5 \mathrm{cde}$ \\
S08 & $4.1 \mathrm{ab}$ & $31.1 \mathrm{~cd}$ & $1.7 \mathrm{bcd}$ & $35.1 \mathrm{de}$ & $1.7 \mathrm{bcd}$ & $36.8 \mathrm{de}$ \\
S09 & $4.5 \mathrm{ab}$ & $36.5 \mathrm{bc}$ & $1.6 \mathrm{~cd}$ & $41.4 \mathrm{bcd}$ & $1.6 \mathrm{bcd}$ & $43.1 \mathrm{bcd}$ \\
S10 & $4.4 \mathrm{ab}$ & $47.8 \mathrm{a}$ & $1.6 \mathrm{bcd}$ & $52.8 \mathrm{a}$ & $1.6 \mathrm{~cd}$ & $54.4 \mathrm{a}$ \\
Average & 4.4 & 37.5 & 1.8 & 42.4 & 1.8 & 44.3 \\
\hline
\end{tabular}

${ }^{\mathrm{z}}$ Values are mean of three independent replications.

${ }^{\mathrm{y}}$ Means followed by different letters within a column are significantly different at $\mathrm{p}<0.05$ by Duncan's multiple range tests.

than those of Ryan et al. (2007), who reported $26 \mathrm{mg} / \mathrm{kg}$ of total tocopherol in Italian millet. Tocopherols content in this study were higher than in barley $(16 \mathrm{mg} / \mathrm{kg})$, buckwheat (46 mg/kg) and maize (13 mg/kg) (Ryan et al., 2007). We observed only two forms of tocotrienol isomers; $a$ - and $\gamma$-tocotrienol in Italian millet landraces and the total tocotrienols content varied from $7.4 \mathrm{mg} / \mathrm{kg}$ (M10) to $11.9 \mathrm{mg} / \mathrm{kg}$ (M09) with an average of $9.2 \mathrm{mg} / \mathrm{kg}$. Total vitamin E content in Italian millet seeds varied from $74.7 \mathrm{mg} / \mathrm{kg}$ in M07 landrace to $101.9 \mathrm{mg} / \mathrm{kg}$ in M01 landrace with an average of $88.3 \mathrm{mg} / \mathrm{kg}$. Compared to
Italian millet, tested sorghum landraces showed somewhat different vitamin $\mathrm{E}$ content pattern in that only 3 vitamin $\mathrm{E}$ isomers could be quantified under our experimental conditions. The $\gamma$-tocopherol, a dominant form of vitamin $E$, varied from $27.1 \mathrm{mg} / \mathrm{kg}$ in S04 to $47.8 \mathrm{mg} / \mathrm{kg}$ in S10 landrace (Table 3). Total vitamin E content which varied from $33.2 \mathrm{mg} / \mathrm{kg}$ (S04) to $54.4 \mathrm{mg} / \mathrm{kg}$ (S10) with an average of $44.3 \mathrm{mg} / \mathrm{kg}$ was lower than that in Italian millet. Except for $\delta$-tocopherol in Italian millet, other vitamin $\mathrm{E}$ isomers showed statistically significant values among the landraces. 
The Contents of Phytosterols, Squalene, and Vitamin E and the Composition of Fatty Acids of Korean Landrace Setaria italica and Sorghum bicolar Seeds

Table 4. Squalene and phytosterols contents in seeds of Italian millet landraces $(\mathrm{mg} / \mathrm{kg})$

\begin{tabular}{cccccc}
\hline \hline Landrace & Squalene & Campesterol & Stigmasterol & B-sitosterol & Total phytosterol \\
\hline M01 & $7.9^{\mathrm{z}} \mathrm{bc}^{\mathrm{y}}$ & $32.2 \mathrm{a}$ & $35.4 \mathrm{bc}$ & $420.2 \mathrm{bcd}$ & $487.9 \mathrm{abc}$ \\
M02 & $7.7 \mathrm{bc}$ & $28.4 \mathrm{a}$ & $32.9 \mathrm{c}$ & $443.1 \mathrm{abcd}$ & $504.4 \mathrm{abc}$ \\
M03 & $9.0 \mathrm{abc}$ & $27.5 \mathrm{a}$ & $45.1 \mathrm{ab}$ & $427.3 \mathrm{abcd}$ & $500.0 \mathrm{abc}$ \\
M04 & $9.4 \mathrm{ba}$ & $31.2 \mathrm{a}$ & $44.6 \mathrm{ab}$ & $427.3 \mathrm{abcd}$ & $503.1 \mathrm{abc}$ \\
M05 & $7.4 \mathrm{ab}$ & $32.7 \mathrm{a}$ & $45.8 \mathrm{ab}$ & $415.7 \mathrm{bcd}$ & $494.1 \mathrm{abc}$ \\
M06 & $7.2 \mathrm{ab}$ & $27.8 \mathrm{a}$ & $37.5 \mathrm{abc}$ & $377.7 \mathrm{~d}$ & $443.0 \mathrm{c}$ \\
M07 & $7.9 \mathrm{ab}$ & $27.3 \mathrm{a}$ & $46.7 \mathrm{a}$ & $403.2 \mathrm{~cd}$ & $477.2 \mathrm{bc}$ \\
M08 & $6.8 \mathrm{c}$ & $25.3 \mathrm{a}$ & $40.2 \mathrm{abc}$ & $453.3 \mathrm{abc}$ & $518.7 \mathrm{abc}$ \\
M09 & $10.2 \mathrm{a}$ & $30.2 \mathrm{a}$ & $43.1 \mathrm{abc}$ & $495.3 \mathrm{a}$ & $568.5 \mathrm{a}$ \\
M10 & $9.2 \mathrm{ab}$ & $24.9 \mathrm{a}$ & $40.4 \mathrm{abc}$ & $482.4 \mathrm{ab}$ & $547.7 \mathrm{bc}$ \\
Average & 8.3 & 28.7 & 41.2 & 434.5 & 504.5 \\
\hline
\end{tabular}

${ }^{\mathrm{z}}$ Values are mean of three independent replications.

${ }^{\mathrm{y}}$ Means followed by different letters within a column are significantly different at $\mathrm{p}<0.05$ by Duncan's multiple range tests.

Table 5. Squalene and phytosterols contents in seeds of sorghum landraces $(\mathrm{mg} / \mathrm{kg})$

\begin{tabular}{cccccc}
\hline \hline Landrace & Squalene & Campesterol & Stigmasterol & $\beta$-sitosterol & Total phytosterol \\
\hline S01 & $78.1^{\mathrm{z}} \mathrm{bc}^{\mathrm{y}}$ & $67.6 \mathrm{c}$ & $59.0 \mathrm{f}$ & $315.7 \mathrm{~cd}$ & $442.3 \mathrm{~cd}$ \\
S02 & $80.1 \mathrm{bc}$ & $69.8 \mathrm{c}$ & $56.8 \mathrm{f}$ & $321.4 \mathrm{~cd}$ & $448.1 \mathrm{~cd}$ \\
S03 & $84.0 \mathrm{~b}$ & $61.1 \mathrm{~cd}$ & $48.2 \mathrm{f}$ & $295.9 \mathrm{~d}$ & $405.2 \mathrm{~d}$ \\
S04 & $62.2 \mathrm{~d}$ & $49.4 \mathrm{~d}$ & $104.1 \mathrm{cde}$ & $321.4 \mathrm{~cd}$ & $474.9 \mathrm{c}$ \\
S05 & $68.7 \mathrm{~d}$ & $54.9 \mathrm{~d}$ & $93.9 \mathrm{e}$ & $307.5 \mathrm{~d}$ & $456.3 \mathrm{~cd}$ \\
S06 & $86.4 \mathrm{~b}$ & $69.9 \mathrm{c}$ & $99.1 \mathrm{de}$ & $329.6 \mathrm{~cd}$ & $498.6 \mathrm{c}$ \\
S07 & $111.3 \mathrm{a}$ & $102.6 \mathrm{a}$ & $120.3 \mathrm{bc}$ & $351.8 \mathrm{bc}$ & $574.7 \mathrm{~b}$ \\
S08 & $115.2 \mathrm{a}$ & $92.4 \mathrm{~b}$ & $123.7 \mathrm{~b}$ & $375.2 \mathrm{~b}$ & $591.2 \mathrm{~b}$ \\
S09 & $83.2 \mathrm{~b}$ & $87.6 \mathrm{~b}$ & $113.0 \mathrm{bcd}$ & $368.1 \mathrm{~b}$ & $568.7 \mathrm{~b}$ \\
S10 & $77.9 \mathrm{bc}$ & $100.1 \mathrm{a}$ & $147.1 \mathrm{a}$ & $472.0 \mathrm{a}$ & $719.2 \mathrm{a}$ \\
Average & 84.7 & 75.5 & 96.5 & 345.9 & 517.9 \\
\hline
\end{tabular}

${ }^{\mathrm{z}}$ Values are mean of three independent replications.

${ }^{\mathrm{y}}$ Means followed by different letters within a column are significantly different at $\mathrm{p}<0.05$ by Duncan's multiple range tests.

\section{Phytosterols content}

The levels of phytosterols (campesterol, stigmasterol and $\beta$-sitosterol) in Italian millet seeds were analyzed. $\beta$-sitosterol, which ranged from $377.7 \mathrm{mg} / \mathrm{kg}$ in M06 to $495.3 \mathrm{mg} / \mathrm{kg}$ in M09, was present in highest quantity comprising about $85 \%$ of total phytosterol content, which was followed by stigmasterol and then campesterol (Table 4). Total phytosterol content in Italian millet landraces varied from 443.0 (M06) to $568.5 \mathrm{mg} / \mathrm{kg}$ (M09) with the average of $504.5 \mathrm{mg} / \mathrm{kg}$. Average quantity of $\beta$-sitosterol in this study was quite similar to the Ryan et al. (2007) and higher than reported by Piironen et al. (2002), however we found lower campesterol $(28.7 \mathrm{mg} / \mathrm{kg})$ and higher stigmasterol $(41.2 \mathrm{mg} / \mathrm{kg})$ that might be due to the genetic differences of Italian millet landraces. Average total phytosterols content of Italian millet was $504.5 \mathrm{mg} / \mathrm{kg}$, which was higher than in maize $(436 \mathrm{mg} / \mathrm{kg})$ and similar to barley $(504 \mathrm{mg} / \mathrm{kg})$ (Ryan et al., 2007). Similar to the Italian millet, sorghum also possessed higher $\beta$-sitosterol compared to campesterol and stigmasterol (Table 5) and sorghum's average contents of campesterol $(75.5 \mathrm{mg} / \mathrm{kg})$ and stigmasterol $(96.5 \mathrm{mg} / \mathrm{kg})$ were higher than those in Italian millet. The phytosterols content in sorghum was similar to Singh et al. (2003) who reported 460 
to $510 \mathrm{mg} / \mathrm{kg}$ of total phytosterols in grain sorghum and higher than in maize and barley (Ryan et al., 2007). Among tested ten sorghum landraces we found exceptionally higher total phytosterols content in $\mathrm{S} 10(719.2 \mathrm{mg} / \mathrm{kg})$ suggesting its superiority than other landraces in terms of phytosterols content. All these results suggest that Italian millet and sorghum can be used as a good source of phytosterols for human diet compared to other cereals for the improvement of human health based upon phytosterols' lowering the serum-cholesterol (Marangoni and Poli, 2010) and anti-carcinogenic activities (de Jong et al., 2003).

\section{Squalene content}

Squalene, a precursor of biosynthetic pathway of phytosterols, content in ten Italian millet landraces varied from $6.8 \mathrm{mg} / \mathrm{kg}$ (M08) to $10.2 \mathrm{mg} / \mathrm{kg}$ (M09) (Table 4), and the average squalene content was $8.3 \mathrm{mg} / \mathrm{kg}$. Compared to the Italian millet, sorghum landraces exhibited higher squalene content in that it varied from $62.2 \mathrm{mg} / \mathrm{kg}$ (S04) to $115.2 \mathrm{mg} / \mathrm{kg}$ (S08) with an average of $84.7 \mathrm{mg} / \mathrm{kg}$ (Table 5). In both crop cases, statistically significant difference could be observed among landraces. The present study showed relatively higher squalene content in sorghum landraces compared to that in barley $(2.0 \mathrm{mg} / \mathrm{kg})$, maize $(16.0 \mathrm{mg} / \mathrm{kg})$, spelt $(20.0 \mathrm{mg} / \mathrm{kg})$ and buckwheat $(19.0$ $\mathrm{mg} / \mathrm{kg}$ ) (Ryan et al., 2007) suggesting sorghum as a good source of squalene.

\section{Fatty acid composition}

Among thirty seven fatty acids screened, seven fatty acids could be quantified in Italian millet. Palmitic (12.3\%), oleic (11.7\%) and linoleic (65.2\%) acids were the major fatty acids comprising over $85 \%$ of total fatty acids (Table 6 ). Other fatty acids were stearic, linolenic, arachidic and behenic acids, which consisted $5.6 \%, 3.4 \%, 1.4 \%$ and $0.5 \%$ of total fatty acids, respectively. The composition of saturated fatty acids (SFA) ranged from $18.3 \%$ to $21.8 \%$, while mono-unsaturated (MUFA) and poly-unsaturated fatty acids (PUFA) were the major forms consisting almost $80 \%$ of total fatty acids in Italian millet seeds. Unlike the case of Italian millet, behenic acid could not be detected in sorghum seeds under our experimental conditions and six fatty acids were quantified. Fatty acids composition of sorghum seeds was quite similar to Italian millet. Sorghum also exhibited palmitic, oleic and linoleic acids as major fatty acids with an average composition of $15.5 \%, 34.9 \%$, and $45.8 \%$ of total fatty acids, respectively, accounting more than $95 \%$ of total fatty acids (Table 7). Similar compositional ratio was also reported by Mehmood et al. (2008). However, sorghum seeds exhibited relatively higher

Table 6. Fatty acid composition in seeds of Italian millet landraces (\%)

\begin{tabular}{|c|c|c|c|c|c|c|c|c|c|c|}
\hline Landrace & $\begin{array}{l}\text { Palmitic } \\
\text { acid } \\
(\mathrm{C} 16: 0)\end{array}$ & $\begin{array}{c}\text { Stearic } \\
\text { acid } \\
(\mathrm{C} 18: 0)\end{array}$ & $\begin{array}{l}\text { Oleic } \\
\text { acid } \\
(\mathrm{C} 18: 1)\end{array}$ & $\begin{array}{c}\text { Linoleic } \\
\text { acid } \\
(\mathrm{C} 18: 2)\end{array}$ & $\begin{array}{c}\text { Linolenic } \\
\text { acid } \\
(\mathrm{C} 18: 3)\end{array}$ & $\begin{array}{c}\text { Arachidic } \\
\text { acid } \\
(\mathrm{C} 20: 0)\end{array}$ & $\begin{array}{l}\text { Behenic } \\
\text { acid } \\
(\mathrm{C} 22: 0)\end{array}$ & $\begin{array}{l}\text { Saturated } \\
\text { fatty acids }\end{array}$ & $\begin{array}{c}\text { Mono- } \\
\text { unsaturated } \\
\text { fatty acid }\end{array}$ & $\begin{array}{c}\text { Poly- } \\
\text { unsaturated } \\
\text { fatty acids }\end{array}$ \\
\hline M01 & $12.19^{\mathrm{z}} \mathrm{ab}^{\mathrm{y}}$ & $6.05 b$ & $12.56 \mathrm{a}$ & $64.50 \mathrm{c}$ & $2.84 \mathrm{e}$ & $1.40 \mathrm{bc}$ & $0.46 \mathrm{bc}$ & $20.10 \mathrm{~b}$ & $12.56 \mathrm{a}$ & $67.34 d$ \\
\hline M02 & $12.13 \mathrm{ab}$ & $6.08 \mathrm{~b}$ & $12.60 \mathrm{a}$ & $64.34 \mathrm{~cd}$ & 2.99de & $1.38 b c$ & $0.48 b c$ & $20.08 b$ & $12.60 \mathrm{a}$ & $67.34 d$ \\
\hline M03 & $12.06 \mathrm{ab}$ & $7.25 \mathrm{a}$ & $11.96 \mathrm{~b}$ & $62.78 \mathrm{e}$ & $3.46 \mathrm{c}$ & $1.78 \mathrm{a}$ & $0.70 \mathrm{a}$ & $21.80 \mathrm{a}$ & $11.96 \mathrm{~b}$ & $66.24 \mathrm{e}$ \\
\hline M04 & $11.84 \mathrm{ab}$ & $4.62 \mathrm{e}$ & $11.19 \mathrm{~cd}$ & $67.02 \mathrm{~d}$ & $3.43 \mathrm{c}$ & $1.32 \mathrm{bcd}$ & $0.58 \mathrm{abc}$ & $18.36 \mathrm{c}$ & $11.19 \mathrm{~cd}$ & $70.45 \mathrm{a}$ \\
\hline M05 & $13.11 \mathrm{a}$ & $4.95 \mathrm{~d}$ & $10.33 \mathrm{e}$ & $65.76 \mathrm{~b}$ & $3.71 \mathrm{abc}$ & $1.49 \mathrm{~b}$ & $0.64 \mathrm{ba}$ & $20.19 b$ & $10.33 \mathrm{e}$ & $69.48 \mathrm{ab}$ \\
\hline M06 & $11.42 \mathrm{~b}$ & 4.80de & $11.45 \mathrm{c}$ & $67.13 a$ & $3.16 \mathrm{~d}$ & $1.46 \mathrm{~b}$ & $0.58 \mathrm{abc}$ & $18.26 \mathrm{c}$ & $11.45 \mathrm{c}$ & $70.29 a$ \\
\hline M07 & $11.80 \mathrm{ab}$ & $4.57 \mathrm{e}$ & $11.30 \mathrm{c}$ & $66.91 \mathrm{a}$ & $3.51 \mathrm{bc}$ & $1.34 \mathrm{bcd}$ & $0.58 \mathrm{abc}$ & $18.28 \mathrm{c}$ & $11.30 \mathrm{c}$ & $70.42 a$ \\
\hline M08 & $11.67 \mathrm{ab}$ & $6.16 b$ & $12.45 \mathrm{a}$ & $65.07 b c$ & $2.98 \mathrm{de}$ & $1.24 \mathrm{bcd}$ & $0.43 b c$ & $19.49 b c$ & $12.45 \mathrm{a}$ & $68.05 \mathrm{~cd}$ \\
\hline M09 & $13.15 \mathrm{a}$ & $6.01 \mathrm{~b}$ & $10.77 \mathrm{~d}$ & $64.84 b c$ & $3.79 \mathrm{ab}$ & $1.04 \mathrm{~d}$ & $0.41 \mathrm{c}$ & $20.61 \mathrm{ab}$ & $10.77 \mathrm{~d}$ & $68.62 \mathrm{bc}$ \\
\hline M10 & $13.13 \mathrm{a}$ & $5.37 \mathrm{c}$ & $12.65 \mathrm{a}$ & 63.39de & $3.88 \mathrm{a}$ & $1.14 \mathrm{~cd}$ & $0.44 b c$ & $20.08 b$ & $12.65 \mathrm{a}$ & $67.27 \mathrm{~d}$ \\
\hline Average & 12.25 & 5.58 & 11.73 & 65.17 & 3.38 & 1.36 & 0.53 & 19.73 & 11.73 & 68.55 \\
\hline
\end{tabular}

${ }^{\mathrm{z}}$ Values are mean of three independent replications.

${ }^{\mathrm{y}}$ Means followed by different letters within a column are significantly different at $\mathrm{p}<0.05$ by Duncan's multiple range tests. 
Table 7. Fatty acid composition in seeds of sorghum landraces (\%)

\begin{tabular}{|c|c|c|c|c|c|c|c|c|c|}
\hline Landrace & $\begin{array}{l}\text { Palmitic acid } \\
\text { (C16:0) }\end{array}$ & $\begin{array}{l}\text { Stearic acid } \\
\text { (C18:0) }\end{array}$ & $\begin{array}{l}\text { Oleic acid } \\
(\mathrm{C} 18: 1)\end{array}$ & $\begin{array}{c}\text { Linoleic } \\
\text { acid } \\
(\mathrm{C} 18: 2)\end{array}$ & $\begin{array}{l}\text { Linolenic } \\
\text { acid } \\
(\mathrm{C} 18: 3)\end{array}$ & $\begin{array}{c}\text { Arachidic } \\
\text { acid } \\
(\mathrm{C} 20: 0)\end{array}$ & $\begin{array}{l}\text { Saturated } \\
\text { fatty acids }\end{array}$ & $\begin{array}{c}\text { Mono- } \\
\text { unsaturated } \\
\text { fatty acid }\end{array}$ & $\begin{array}{c}\text { Poly- } \\
\text { unsaturated } \\
\text { fatty acids }\end{array}$ \\
\hline S01 & $15.22^{z} \operatorname{cde}^{y}$ & $1.82 \mathrm{abc}$ & $35.71 \mathrm{~b}$ & $45.27 \mathrm{~cd}$ & $1.51 \mathrm{de}$ & $0.32 \mathrm{ab}$ & $17.36 \mathrm{bc}$ & $35.71 \mathrm{~b}$ & $46.78 \mathrm{~cd}$ \\
\hline S02 & $14.90 \mathrm{de}$ & $1.91 \mathrm{bc}$ & $36.03 \mathrm{~b}$ & $44.82 \mathrm{~d}$ & $1.78 \mathrm{abc}$ & $0.33 \mathrm{ab}$ & $17.15 \mathrm{bc}$ & $36.03 b$ & $46.62 \mathrm{~d}$ \\
\hline S03 & $14.63 \mathrm{e}$ & $2.02 \mathrm{a}$ & $35.11 \mathrm{c}$ & $45.77 \mathrm{bc}$ & $1.82 \mathrm{ab}$ & $0.45 \mathrm{a}$ & $17.10 \mathrm{bc}$ & $35.11 \mathrm{c}$ & $47.59 b$ \\
\hline S04 & $16.75 \mathrm{a}$ & $1.69 \mathrm{bcd}$ & $33.78 \mathrm{~d}$ & $45.80 \mathrm{bc}$ & $1.69 \mathrm{bcd}$ & $0.20 \mathrm{~b}$ & $18.91 \mathrm{a}$ & $33.78 \mathrm{~d}$ & $47.08 \mathrm{~b}$ \\
\hline S05 & $15.82 \mathrm{bd}$ & $1.84 \mathrm{abc}$ & $29.52 \mathrm{e}$ & $51.10 \mathrm{a}$ & $1.41 \mathrm{e}$ & $0.24 \mathrm{~b}$ & $17.90 \mathrm{ab}$ & $29.52 \mathrm{e}$ & $52.50 \mathrm{a}$ \\
\hline S06 & $15.51 \mathrm{bcd}$ & $1.86 \mathrm{abc}$ & $34.86 a$ & $45.58 \mathrm{bcd}$ & $1.79 \mathrm{abc}$ & $0.33 \mathrm{ab}$ & $17.70 \mathrm{~b}$ & $34.86 \mathrm{c}$ & $47.37 \mathrm{bc}$ \\
\hline S07 & $16.14 b$ & $1.60 \mathrm{~cd}$ & $37.77 \mathrm{a}$ & $42.08 \mathrm{e}$ & $1.99 \mathrm{a}$ & $0.31 \mathrm{ab}$ & $18.05 \mathrm{ab}$ & $37.77 \mathrm{a}$ & $44.07 \mathrm{e}$ \\
\hline S08 & $15.39 \mathrm{bcde}$ & 1.91ab & $34.71 \mathrm{c}$ & $46.20 \mathrm{~b}$ & $1.54 \mathrm{de}$ & $0.27 \mathrm{~b}$ & $17.60 \mathrm{bc}$ & $34.71 \mathrm{c}$ & $47.49 b$ \\
\hline S09 & $15.59 \mathrm{bcd}$ & $1.56 \mathrm{~d}$ & $35.97 \mathrm{~b}$ & $44.92 \mathrm{~d}$ & $1.58 \mathrm{cde}$ & $0.27 b$ & $17.42 \mathrm{bc}$ & $35.97 \mathrm{~b}$ & $46.50 \mathrm{~d}$ \\
\hline S10 & $14.62 \mathrm{e}$ & $1.74 \mathrm{bcd}$ & $35.72 b$ & $45.92 \mathrm{bc}$ & $1.66 \mathrm{bcd}$ & $0.27 b$ & $16.65 \mathrm{c}$ & $35.72 b$ & $47.58 b$ \\
\hline Average & 15.46 & 1.79 & 34.92 & 45.75 & 1.68 & 0.30 & 17.58 & 34.94 & 47.36 \\
\hline
\end{tabular}

${ }^{\mathrm{z}}$ Values are mean of three independent replications.

${ }^{\mathrm{y}}$ Means followed by different letters within a column are significantly different at $\mathrm{p}<0.05$ by Duncan's multiple range tests.

Table 8. Correlationships among phytonutrients in Italian millet seeds

\begin{tabular}{|c|c|c|c|c|c|c|c|c|c|c|c|c|c|c|c|}
\hline Phytonutrients & $\begin{array}{c}\text { Stearic } \\
\text { acid }\end{array}$ & $\begin{array}{c}\text { Oleic } \\
\text { acid }\end{array}$ & $\begin{array}{l}\text { Linoleic } \\
\text { acid }\end{array}$ & $\begin{array}{c}\text { Arachidic } \\
\text { acid }\end{array}$ & $\begin{array}{c}\text { Linolenic } \\
\text { acid }\end{array}$ & $\begin{array}{c}\text { Behenic } \\
\text { acid }\end{array}$ & Squalene & $\begin{array}{l}\text { Campe- } \\
\text { sterol }\end{array}$ & $\begin{array}{c}\text { Stigma- } \\
\text { sterol }\end{array}$ & $\begin{array}{c}\beta \text {-sito- } \\
\text { sterol }\end{array}$ & $\begin{array}{l}\text { a-toco- } \\
\text { pherol }\end{array}$ & $\begin{array}{l}\text { a-toco- } \\
\text { trienol }\end{array}$ & $\begin{array}{l}\gamma \text {-toco- } \\
\text { pherol }\end{array}$ & $\begin{array}{l}\gamma \text {-toco- } \\
\text { trienol }\end{array}$ & $\begin{array}{l}\delta \text {-toco- } \\
\text { pherol }\end{array}$ \\
\hline Palmitic acid & -0.02 & -0.338 & $-0.491 * *$ & -0.313 & $0.552^{* *}$ & 0.061 & $0.593 * *$ & 0.332 & 0.237 & $0.444 * *$ & -0.185 & 0.269 & 0.056 & $0.378^{*}$ & 0.096 \\
\hline Stearic acid & & $0.475^{* *}$ & $-0.766^{* *}$ & 0.272 & -0.238 & -0.106 & 0.05 & -0.129 & -0.178 & 0.18 & -0.339 & $0.636^{* *}$ & 0.309 & 0.212 & 0.046 \\
\hline Oleic acid & & & $-0.443^{*}$ & 0.024 & $-0.521 * *$ & -0.352 & -0.247 & -0.314 & $-0.524 * *$ & 0.047 & $-0.449 *$ & 0.155 & $0.419^{*}$ & $-0.555^{* *}$ & -0.052 \\
\hline Linoleic acid & & & & -0.137 & -0.129 & 0.003 & -0.353 & 0.004 & 0.065 & $-0.381^{*}$ & $0.366^{*}$ & $-0.543 * *$ & -0.29 & -0.17 & -0.075 \\
\hline Arachidic acid & & & & & -0.223 & $0.704 * *$ & -0.161 & 0.16 & 0.187 & -0.272 & $0.433^{*}$ & 0.096 & 0.053 & 0.17 & 0.238 \\
\hline Linolenic acid & & & & & & 0.051 & $0.453^{* *}$ & -0.089 & $0.455^{*}$ & 0.172 & 0.228 & -0.199 & $-0.498^{* *}$ & 0.338 & -0.069 \\
\hline Behenic acid & & & & & & & 0.242 & $0.395^{* *}$ & $0.383 *$ & -0.068 & $0.560 * *$ & -0.169 & -0.279 & 0.234 & 0.012 \\
\hline Squalene & & & & & & & & $0.399 * *$ & 0.31 & $0.524 * *$ & 0.247 & 0.142 & -0.107 & $0.530^{* *}$ & 0.003 \\
\hline Campesterol & & & & & & & & & 0.282 & $0.582^{* *}$ & 0.155 & 0.044 & 0.296 & $0.384^{*}$ & 0.104 \\
\hline Stigmasterol & & & & & & & & & & 0.239 & $0.394 *$ & -0.198 & -0.375 & $0.367^{*}$ & -0.025 \\
\hline$\beta$-sitosterol & & & & & & & & & & & -0.235 & 0.124 & 0.262 & 0.223 & -0.151 \\
\hline a-tocopherol & & & & & & & & & & & & -0.181 & -0.385 & $0.392 *$ & 0.294 \\
\hline a-tocotrienol & & & & & & & & & & & & & 0.481 & $0.439 *$ & $0.546^{* *}$ \\
\hline$\gamma$-tocopherol & & & & & & & & & & & & & & 0.111 & 0.291 \\
\hline$\gamma$-tocotrienol & & & & & & & & & & & & & & & 0.264 \\
\hline
\end{tabular}

$*, * *$ Correlation is significant at $\mathrm{p}<0.05$ and $\mathrm{p}<0.01$ levels, respectively.

composition of oleic acid (34.9\%) compared to Italian millet. The other fatty acids were stearic acid (1.8\%), linolenic acid $(1.7 \%)$ and arachidic acids $(0.3 \%)$. In the present study, no special landrace was noticed having exceptionally higher composition of fatty acids than other. The saturated fatty acid of sorghum seeds ranged from $16.7 \%$ (S10) to $18.9 \%$ (S04) with an average of $17.6 \%$. Similar to the Italian millet, sorghum seeds also exhibited about $80 \%$ of total unsaturated fatty acids. All tested sorghum landraces showed over $44.1 \%$ PUFA. High composition of unsaturated fatty acid in both Italian millet and sorghum seeds suggested higher health beneficial value of these crops since these unsaturated fatty acids may decrease the blood cholesterol levels (Hargrove et al., 2001), modulate immune function, and decrease susceptibility of 
Korean J. Plant Res. 26(6) : 663 672 (2013)

Table 9. Correlationships among phytonutrients in sorghum seeds

\begin{tabular}{|c|c|c|c|c|c|c|c|c|c|c|c|c|}
\hline Phytonutrients & $\begin{array}{c}\text { Stearic } \\
\text { acid }\end{array}$ & $\begin{array}{l}\text { Oleic } \\
\text { acid }\end{array}$ & $\begin{array}{c}\text { Linoleic } \\
\text { acid }\end{array}$ & $\begin{array}{c}\text { Arachidic } \\
\text { acid }\end{array}$ & $\begin{array}{c}\text { Linolenic } \\
\text { acid }\end{array}$ & Squalene & $\begin{array}{c}\text { Campe- } \\
\text { sterol }\end{array}$ & $\begin{array}{c}\text { Stigma- } \\
\text { sterol }\end{array}$ & $\begin{array}{c}\beta \text {-sito- } \\
\text { sterol }\end{array}$ & $\begin{array}{l}\text { a-toco- } \\
\text { pherol }\end{array}$ & $\begin{array}{l}\text { a-toco- } \\
\text { trienol }\end{array}$ & $\begin{array}{l}\text { V-toco- } \\
\text { pherol }\end{array}$ \\
\hline Palmitic acid & $-0.446^{*}$ & -0.228 & -0.07 & -0.157 & 0.056 & -0.171 & -0.186 & 0.119 & -0.29 & -0.015 & 0.16 & $-0.760 * *$ \\
\hline Stearic acid & & -0.241 & 0.302 & 0.068 & -0.073 & -0.112 & $-0.44^{*}$ & $-0.501^{*}$ & -0.317 & -0.194 & -0.279 & 0.163 \\
\hline Oleic acid & & & $-0.942 * *$ & 0.23 & $0.542 * *$ & $0.402 *$ & $0.561 * *$ & 0.079 & 0.268 & -0.279 & $0.537 * *$ & 0.285 \\
\hline Linoleic acid & & & & -0.293 & $-0.668 * *$ & -0.352 & -0.452 & -0.037 & -0.106 & 0.335 & $-0.592 * *$ & -0.056 \\
\hline Arachidic acid & & & & & $0.425^{*}$ & 0.06 & -0.111 & $-0.381^{*}$ & -0.331 & -0.364 & 0.045 & 0.238 \\
\hline Linoenic acid & & & & & & 0.29 & 0.181 & -0.036 & -0.017 & -0.261 & $0.454 *$ & 0.016 \\
\hline Squalene & & & & & & & $0.532 * *$ & 0.333 & 0.239 & -0.285 & $0.401 *$ & -0.106 \\
\hline Campesterol & & & & & & & & $0.611 * *$ & $0.776^{* *}$ & 0.091 & $0.518^{* *}$ & 0.300 \\
\hline Stigmasterol & & & & & & & & & $0.727 * *$ & 0.083 & 0.130 & -0.126 \\
\hline$\beta$-sitosterol & & & & & & & & & & 0.252 & 0.083 & $0.406^{*}$ \\
\hline a-tocopherol & & & & & & & & & & & 0.073 & 0.36 \\
\hline a-tocotrienol & & & & & & & & & & & & 0.041 \\
\hline
\end{tabular}

*, ** Correlation is significant at $\mathrm{p}<0.05$ and $\mathrm{p}<0.01$ levels, respectively.

oxidation of LDL and improve the fluidity of HDL (Villa et al., 2002). These results suggest that sorghum and Italian millet seeds may serve as good sources of healthy food due to high ratio of unsaturated fatty acids.

\section{Correlationship among phytonutrients}

Statistical correlationships among phytonutrients (vitamin E, squalene, phytosterols and fatty acids) were analyzed for both Italian millet and sorghum seeds. In the case of Italian millet seeds, squalene showed positive correlationship with campesterol $\left(\mathrm{r}=0.399^{* *}\right), \beta$-sitosterol $\left(\mathrm{r}=0.524^{* *}\right)$ and $\mathrm{a}$ -tocopherol $\left(\mathrm{r}=0.530^{* *}\right)$ as well as with palmitic acid $(\mathrm{r}=$ $\left.0.593^{* *}\right)$ and linolenic acid $\left(\mathrm{r}=0.453^{* *}\right)($ Table 8$)$. Among the vitamin $\mathrm{E}$ isomers a-tocotrienol showed somewhat high positive correlationship with $\delta$-tocopherol $\left(\mathrm{r}=0.546^{* *}\right)$. Among phytosterols $\beta$-sitosterol showed positive correlationship with campesterol $\left(\mathrm{r}=0.582^{* *}\right)$, but not with stigmasterol $(\mathrm{r}=$ $\left.0.239^{\mathrm{NS}}\right)$. Sorghum seeds exhibited somewhat different correlationship among phytonutrients compared to Italian millet in that squalene showed positive correlationship only with campesterol $\left(\mathrm{r}=0.532^{* *}\right)$ and $\beta$-sitosterol exhibited correlationship with both campesterol $\left(\mathrm{r}=0.776^{* *}\right)$ and stigmasterol $\left(\mathrm{r}=0.727^{* *}\right)$ (Table 9). The higher positive correlationships among phytosterols and squalene observed in both Italian millet and sorghum may result from the facts that phytosterols are produced by using squalene as a preceding material for their synthesis (Piironen et al., 2000 and references there in).

\section{Conclusion}

This study shows that both Italian millet and sorghum are a rich source of phytonutrients, justifying the dietary food status of these crops in various countries. In both sorghum and Italian millet landraces, $\gamma$-tocopherol was present in highest quantity among vitamin $\mathrm{E}$ isomers and $\beta$-sitosterol was the major phytosterol. The presence of higher content of vitamin $\mathrm{E}$ and phytosterol in Italian millet and sorghum compared to other cereal crops may enrich their nutritional value as a cereal crop. The major fatty acids were linoleic (C18:2n6c), oleic (C18:1n9c) and palmitic (C16:0) acids. High composition of such unsaturated fatty acids suggested sorghum and Italian millet as healthy food source. Among tested ones, sorghum landrace S10 and Italian millet landrace M09 exhibited superiority in term of phytochemicals suggesting these two landraces as a good gene source for further breeding programs.

\section{Acknowledgement}

This research was supported by Soonchunhyang University Research Grant. 


\section{Literature Cited}

Aguilera, Y., M.E. Dorado, F.A. Prada, J.J. Martinez, A. Quesada and V. R. Gutierrez. 2005. The protective role of squalene in alcohol damage in the chick embryo retina. Exp. Eye Res. 80:535-543.

Awika, J.M. and L.W. Rooney. 2004. Sorghum phytochemicals and their potential impact on human health. Phytochemistry 65:1199-1221.

Bhandari, S.R., S. Basnet, K.H. Chung, K.H. Ryu and Y.S. Lee. 2012. Comparisons of nutritional and phytochemical property of genetically modified CMV-resistant red pepper and its parental cultivar. Hort. Environ. Biotechnol. 53:151-157.

Chethan, S. and N.G. Malleshi. 2007. Finger millet polyphenols: characterization and their nutraceutical potential. Am. J. Food Technol. 2:582-592.

Choi, Y. and J. Lee. 2009. Antioxidant and antiproliferative properties of tocotrienol-rich fraction from grape seeds. Food Chem. 114:1386-1390.

de Jong, A., J. Plat and R.P. Mensink. 2003. Metabolic effects of plant sterols and stanols. J. Nutr. Biochem. 14:362-369.

Duodu, K.G., J.R.N. Taylor, P.S. Belton and B.R. Hamaker. 2003. Factors affecting sorghum protein digestibility. J. Cereal Sci. 38:117-131.

Dykes, L. and L.W. Rooney. 2006. Sorghum and millet phenols and antioxidants. J. Cereal Sci. 44:236-251.

FAO. 2011. FAOSTAT ProdStat database, yearly production. URL: $<$ http://faostat.fao.org $>$.

Hargrove, R.L., T.D. Etherton, T.A. Pearson, E.H. Harrison and P.M. Kris-Etherton. 2001. Low-fat and high monounsaturated fat diets decrease human low density lipoprotein oxidative susceptibility in vitro. J. Nutr. 131:1758-1763.

Kamal-Eldin, A. and L.A. Appelqvist. 1996. The chemistry and antioxidant properties of tocopherols and tocotrienols. Lipids 31:671-701.

Kim, J.K., N.H. Kim, J.K. Bang, B.K. Lee, C.B. Park and B.H. Lee. 2000. Fatty acid composition analysis of major oil crops by one step extraction/methylation method. Korean J. Crop Sci. 45:211-215.

Knekt, P., A. Reunanen, R. Jarvinen, R. Seppanen, M. Heliovaara and A. Aromaa. 1994. Antioxidant vitamin intake and coronary mortality in a longitudinal population study. Am. J. Epidemiol. 139:1180-1189.

Kohno, Y., Y. Egawa, S. Itoh, S. Nagaoka, M. Takahashi and K.
Mukai. 1995. Kinetic study of quenching reaction of singlet oxygen and scavenging reaction of free radicals by squalene in n-butanol. Biochem. Biophys. Acta 1256:52-56.

Kushi, L.H., A.R. Folsom, R.J. Prineas, P.J. Mink, Y. Wu and R.M. Bostick. 1996. Dietary antioxidant vitamins and death from coronary heart disease in postmenopausal women. N. Engl. J. Med. 334:1156-1162.

Marangoni, F. and A. Poli. 2010. Phytosterols and cardiovascular health. Pharmacol. Res. 61:193-199.

Mehmood, S., I. Orhan, Z. Ahsan, S. Aslan and M. Gulfraz. 2008. Fatty acid composition of seed oil of different Sorghum bicolor varieties. Food Chem. 109:855-859.

Moreda, W., M.C. Perez-Camino and A. Cert. 2001. Gas and liquid chromatography of hydrocarbons in edible vegetable oils. J. Chromatogr. A 936:159-171.

Ohkatsu, Y., T. Kajiyama and Y. Arai. 2001. Antioxidant activities of tocopherols. Polym. Degrad. Stab. 72:303-311.

O'Kennedy, M.M., A. Grootboom and P.R. Shewry. 2006. Harnessing sorghum and millet biotechnology for food and health. J. Cereal Sci. 44:224-235.

Ostlund, R.E. Jr. 2004. Phytosterols and cholesterol metabolism. Curr. Opin. Lipidol. 15:37-41.

O’Sullivan, L., J.A. Woods and N.M. O'Brien. 2002. Squalene but not n-3 fatty acids protect against hydrogen peroxideinduced sister chromatid exchanges in Chinese hamster V79 cells. Nutr. Res. 22:847-857.

Park, K.Y., C.S. Kang, Y.S. Lee, Y.H. Lee and Y.S. Lee. 2004. Tocotrienol and tocopherol content in various plant seeds. Korean J. Crop Sci. 49:207-210.

Pathak, P., S. Srivastava and S. Grover. 2000. Development of food products based on millet, legumes and fenugreek seeds and their suitability in the diabetic diet. Int. J. Food Sci. Nutr. 51:409-414.

Piironen, V., D.G. Lindsay, T.A. Miettinen, J. Toivo and A.M. Lampi. 2000. Plant sterols: biosynthesis, biological function and their importance to human nutrition. J. Sci. Food Agric. 80:939-966.

Piironen, V., J. Toivo and A.M. Lampi. 2002. Plant sterols in cereals and cereal products. Cereal Chem. 79:148-154.

Qureshi, A.A., B.A. Bradlow, L. Brace, J. Manganello, D.M. Peterson, B.C. Pearce, J.J. Wright, A. Gapor and C.E. Elson. 1995. Response of hypercholesterolemic subjects to administration of tocotrienols. Lipids 30:1171-1177.

Ryan, E., K. Galvin, T.P. O'Connor, A.R. Maguire and N.M. O’Brien. 2007. Phytosterol, squalene, tocopherol content 
and fatty acid profile of selected seeds, grains, and legumes. Plant Foods Hum. Nutr. 62:85-91.

Senthilkumar, S., T. Devaki, B.M. Manohar and M.S. Babu. 2006. Effect of squalene on cyclophosphamide-induced toxicity. Clin. Chim. Acta 364:335-342.

Singh, V., R.A. Moreau and K.B. Hicks. 2003. Yield and phytosterol composition of oil extracted from grain sorghum and its wet-milled fractions. Cereal Chem. 80:126-129.

Smith, T.J. 2000. Squalene: potential chemopreventive agent. Expert. Opin. Investig. Drugs 9:1841-1848.

Tucker, J.M. and D.M. Townsend. 2005. Alpha-tocopherol: roles in prevention and therapy of human disease. Biomed. Pharmacother. 59:380-387.

Villa, B., L. Calabresi, G. Chiesa, P. Rise, C. Galli and C.R. Sirtori. 2002. Omega-3 fatty acid ethyl esters increase heart rate variability in patients with coronary disease. Pharmacol. Res. 45:475-478.

Wada, S., Y. Satomi, M. Murakoshi, N. Noguchi, T. Yoshikawa and H. Nishino. 2005. Tumor suppressive effects of tocotrienol in vivo and in vitro. Cancer Lett. 229:181-191.

Weihrauch, J.L. and J.M. Gardner. 1978. Sterol content of foods of plant origin. J. Am. Diet. Assoc. 73:39-47.

(Received 20 August 2013 ; Revised 22 November 2013 ; Accepted 23 November 2013) 